\title{
Apple II/FIRST-Corvus networks
}

\author{
BEVERLY MARSHALL-GOODELL and I. GORMEZANO \\ University of lowa, lowa City, Iowa
}

\begin{abstract}
Our Apple II/FIRST hardware-software configuration is a cost-effective, powerful, and efficient system for experimental control, data collection, and signal analysis of research in conditioning, neurophysiology, psychopharmacology, and psychophysiology. The present report describes the enhancement of our data-processing capabilities by the addition of peripheral hardware configured to form Corvus multiplexer networks. These networks have transformed our array of Apple II/ FIRST systems into multiprocessor facilities possessing the data-processing power and flexibility of far larger and more costly mainframe systems while retaining the benefits of the markedly reduced cost, great flexibility, and high processing speed of microprocessor-based systems.
\end{abstract}

Our several years of experience with the Apple II/ FIRST system (Scandrett \& Gormezano, 1980) have revealed it to be an admirably cost-effective, powerful, and flexible system for experimental control, data collection, and signal analysis of research in conditioning (Johnson, 1981; Marshall-Goodell, Schreurs, \& Gormezano, 1982), neurophysiology (Schreurs, Gormezano, \& Harvey, 1983), psychopharmacology (Harvey, Gormezano, \& Cool-Hauser, 1983), and psychophysiology (MarshallGoodell, Gormezano, Scandrett, \& Cacioppo, 1981). However, the characteristically limited data-storage and data-communication capabilities of microprocessor-based systems have frequently served to restrict the Apple II/ FIRST's large-scale data-processing applications to cumbersome and time-consuming procedures. The purpose of the present paper is to describe a major augmentation in the system's data-processing capabilities by the addition of peripheral hardware configured to form Corvus multiplexer networks.

\section{THE OPERATING SUBSYSTEMS}

\section{Apple II/FIRST}

Scandrett and Gormezano (1980) described the Apple II/FIRST hardware-software configuration interfaced to each of our three conditioning laboratories. Briefly, each system consists of an Apple II or Apple II + with 48K bytes of memory, a Microsoft 16K byte extended memory (RAM) card, video monitor, Apple II floppy disk drive, and a FIRST language card containing a floating-point processor (AM9511), a programmable counter/timer (8253) providing a real-time clock, two programmable peripheral interfaces (8255), each providing a 24-bit digital input and/or output (I/O) buffer, and a 16-channel

The hardware and software development presented in this report was supported, in part, by NSF Grants BNS 76-8561, 80-05907, and 8309826 and NIMH Grant MH 16841 . We also gratefully acknowledge WGN Enterprises, Inc., for providing office facilities. Requests for reprints should be addressed to I. Gormezano, Department of Psychology, University of Iowa, Iowa City, IA 52242. 8-bit analog-to-digital (A/D) converter. FIRST, resident in $13.8 \mathrm{~K}$ bytes in the Apple II, is a flexible dictionarybased language tailored for the most efficient use of a 48K byte Apple II. FIRST's flexibility is derived from the ability to define new words as combinations of previously defined words, and, thus, any particular program constitutes a customized extension of the FIRST language dictionary. FIRST has been designed for very high processing speed by making extensive use of the AM9511 floating-point processor and the firmware facilities present in the Apple II read-only memory (ROM). Moreover, FIRST completely supercedes the functions usually provided by the Applesoft BASIC language processor, and is typically 10 times faster than Apple integer BASIC for 16-bit arithmetic and 12-70 times faster than Applesoft floating-point BASIC for 32-bit floating-point arithmetic.

Apple II/FIRST's experimental-control and data-acquisition functions are accomplished by using variations of a generalized conditioning program written in FIRST. Stimulus control is carried out by storing or writing output control bits into programmable peripheral interfaces. A programmable interval timer controls the presentation of trial and intertrial stimuli and rate of mulitplexed A/D conversion of response signals (typically every $2-5 \mathrm{msec}$ ). During the intertrial interval, the program displays the digitized response topography for each subject successively. Then, the five dependent variable measures (e.g., response latency, response amplitude) extracted from each response topography for all subjects on that trial are displayed (see Johnson, 1981, and Scandrett \& Gormezano, 1980). After each conditioning trial, the digitized response topography data and the five extracted dependent variable measures are stored on separate floppy disks. Our experiments typically involved 3-10 independent groups $(n=8-12)$, each receiving 5-20 daily sessions of 50-120 trials. In a single session, the trial-by-trial analysis of response topography (for 8-12 subjects) yields 300$1,000 \mathrm{~K}$ bytes of data, whereas that for the dependent variable measures involves $20-50 \mathrm{~K}$ bytes. Accordingly, for any single experiment, $3-10 \mathrm{M}$ bytes of data must be logged onto as many as 25-75 floppy disks, which then 
serve as the input to subsequent data-processing programs (cf. Johnson, 1981; Scandrett \& Gormezano, 1980). Unfortunately, on occasion, portions of these data have been lost through transcribing infidelities. Accordingly, it has become increasingly apparent that our floppy disk system has not only limited our data processing to cumbersome and time-consuming procedures, but has also placed the data at risk.

\section{Corvus Disk}

To overcome the above limitations to the Apple II/ FIRST's large-scale data-processing capabilities, we acquired and interfaced a $10 \mathrm{M}$ byte Corvus mass-storage disk system to one of our laboratories. Briefly, the system consists of a Winchester disk drive with Corvus intelligent disk controller uses a Z-80 processor with $16 \mathrm{~K}$ bytes of RAM for very accurate, high-speed disk access, and its associated Constellation software. The Corvus intelligent disk controller uses a Z-80 processor with $16 \mathrm{~K}$ bytes of RAM for very accurage, high-speed disk access, and the Corvus/Apple II interface and associated controller provide a buffered parallel I/O capable of transferring data at a rate of over $50 \mathrm{~K}$ bytes $/ \mathrm{sec}$. The system's firmware, as contained in $2 \mathrm{~K}$ byte ROM, provides for data transfer and management of disk space. Finally, the Corvus Constellation software provides utility programs for management of disk space into smaller units, called volumes, as well as programs to format the Corvus disk by volumes and to copy between Corvus volumes and floppy disks.

\section{Corvus Mirror}

The $10 \mathrm{M}$ byte Corvus disk system readily permitted our Apple II/FIRST system to address the on-line and off-line data-processing demands of one laboratory. Moreover, by judicious time scheduling, the data collected on floppy disks in our other two conditioning laboratories could be transferred to the $10 \mathrm{M}$ byte Corvus disk for rapid and efficient signal analysis and data processing. However, a quantum leap in these data-processing capabilities was realized by our incorporating a Corvus Mirror for converting the digital contents on the Corvus disk to a video signal for storage on video cassettes. In the Mirror's operation, the firmware resident in the Corvus Z-80 disk controller provides an intelligent interface between the Apple II/FIRST system and a video cassette. Our microcomputer can then specify one of four operations to the Mirror: Backup, Restore, Verify, and Identify. The Backup operation can, for example, transfer the entire contents of the $10 \mathrm{M}$ byte Corvus disk to a video cassette in approximately $15 \mathrm{~min}$, whereas, the Restore operation transfers information from the cassette directly to the Corvus disk in the same time. Information is stored on the video cassette with fourfold redundancy to facilitate error recovery. The Verify operation uses the errordetection hardware in the Mirror and the availability of redundant images to confirm the readability of the recorded data. The Identify operation reads only the image header containing labels of the contents as specified at the time the image was recorded, and is used in locating records. The remote-control capabilities of our Panasonic (Model NV-8200) recorder provide easy access to the more than $35 \mathrm{M}$ byte capacity of a standard 2-h VHS video cassette: A particular file of a volume anywhere on the cassette can be retrieved in minutes. In sum, we have found that the Mirror provides a reliable, efficient, and cost-efficient means of long-term storage and retrieval of our data and programs.

\section{NETWORKS}

The single-user Apple II/FIRST-Corvus disk-Mirror configuration has been in use in our laboratory for several years. However, recently, with the addition of a Corvus multiplexer, the configuration was converted into a multiuser system. Each of our four independent Apple II/ FIRST systems was connected in a star configuration with a Corvus host multiplexer at the center to form a network. Within the network, the Corvus host multiplexer can poll up to eight computers in a round-robin fashion. The firmware for the Corvus multiplexer network, resident in the Corvus disk controller, provides direct communication paths between the microcomputers and controls access to shared volumes. The communication channels to each computer are polled serially at a rate that permits virtually simultaneous use of shared areas of the Corvus disk. Since the sharing of common data and programs by separate microcomputers can be dangerous, we have utilities capable of locking areas on the Corvus disk for read-only use or for access by only one computer at a time.

Because the Corvus disk subsystem is the keystone to

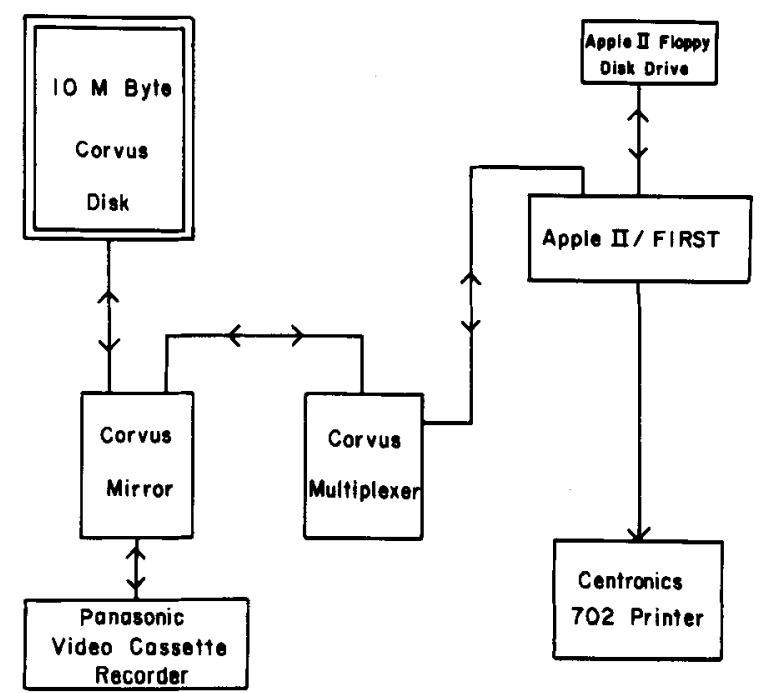

Figure 1. A stand-alone Apple II/FIRST-Corvus network featuring a 10M byte Corvus hard disk (Model 11AP), Corvus Mirror and attached Panasonic video cassette recorder (Model NV-8200), and Corvus Multiplexer. Although the Corvus Multiplexer can accommodate up to eight attached microprocessors, only a single Apple II/FIRST system, including an Apple II floppy disk drive and a Centronics (Model 702) line printer, is shown. 


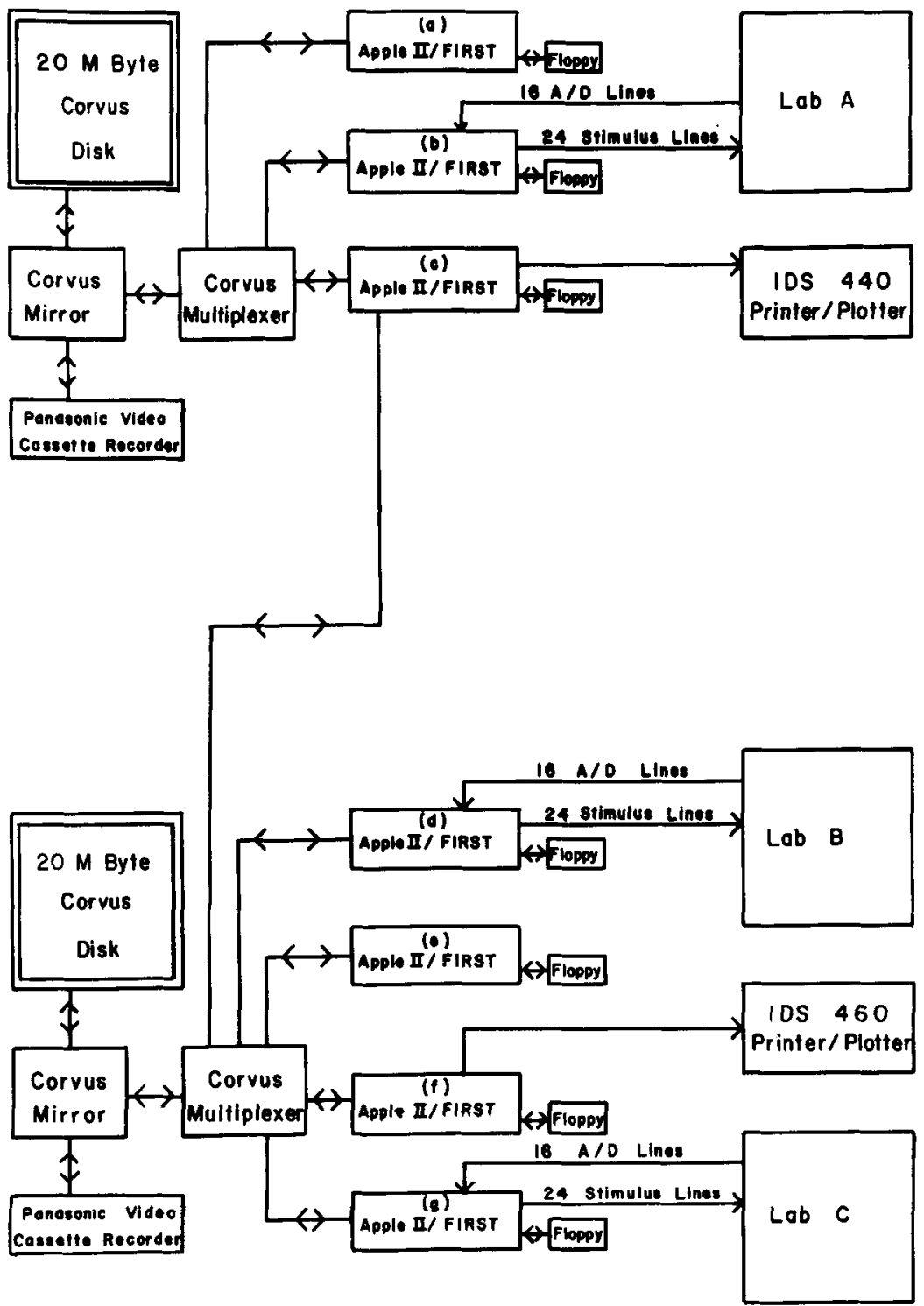

Figure 2. Multiple Apple II/FIRST-Corvus networks featuring two 20M byte Corvus hard disks (Model 20MB, Rev. B), each with a Corvus Mirror, Panasonic video cassette recorder (Model NV-8200), and Corvus Multiplexer. The top network is configured with three Apple I/FIRST systems, one of which (b) is interfaced to Conditioning Lab A for stimulus control and data acquisition, and, of the two others (a and c) that are available for off-line data processing, one (c) is interfaced to an IDS printer/plotter (Paper Tiger Model 44). The bottom network has an array of four Apple II/FIRST systems. One of the systems (d) is interfaced to Conditioning Lab B and other (g) to Conditioning Lab C. Of the other two Apple H/FIRST systems that are available for data processing, one (f) is interfaced to an IDS printer/plotter (Paper Tiger Model 460), and the other (e) is interfaced, via (c), to both networks to permit direct transfer of information between the two Corvus disks.

the Corvus multiplexer network, occasional malfunctions completely disrupted the operation of all three of our conditioning laboratories and the stand-alone data-processing system. These breakdowns, although infrequent, were extremely costly, since the subjects and data from a series of experimental runs, and often an entire experiment, had to be discarded. Therefore, we sought to eliminate the vulnerability of our entire facilities to malfunctions of a critical component by establishing several interchangeable networks. As the budget permitted, we gradually acquired the additional Corvus disks, Mirrors, and multiplexers for establishing three networks. One network, depicted in Figure 1, utilizes the 10M byte Corvus disk system to service our Apple II/FIRST stand-alone dataanalysis system. The other two networks service our three conditioning systems and, as depicted in Figure 2, are each 
configured around separate 20M byte Corvus disks. As can be seen in Figure 2, one network services Lab A, and the other network Labs B and C; and an Apple II/FIRST system, interfaced to both $20 \mathrm{M}$ byte Corvus disks, permits direct Corvus-to-Corvus transfers. By storing identical software on all three Corvus disks, each can be readily employed as a backup system. Although Figures 1 and 2 depict the typical configuration of our networks, the Corvus multiplexer readily permits the reallocation of any Apple II/FIRST microprocessor to another network to bring additional processing power to any particular application.

\section{SOFTWARE ENHANCEMENT}

The successive stages of hardware development in the evolution of our networks were undertaken principally to address limitations imposed by the floppy disk system on the large-scale data-processing capabilities of our array of Apple II/FIRST microprocessors. Nevertheless, we have also substantially affected efficiency in utilizing FIRST. With initial addition of a Corvus disk to the Apple II/FIRST system, our software resident on the Corvus disk consisted of copies of a multitude of floppy disk programs, with no central mechanism for loading programs from their diverse locations. However, the opportunity for almost immediate access to multiple programs for performing similar operations led to the development of a sophisticated software-management system to assure uniformity in new software development and to facilitate access to existing programs. A marked enhancement in software management resulted from the development of a menu system in which the operator loads an individual program by selecting from among a list of options displayed on the monitor. The initial "Master Menu" has options for selecting experimental-control/ data-collection, signal-analysis, data-processing, and utility programs (e.g., floppy-to-Corvus disk and Corvusto-Corvus disk transfers, video storage and retrieval). In addition, the Master Menu provides access to a Reference Manual for FIRST and a volume-by-volume directory of the Corvus disk's contents. Subsequent menus displayed on the monitor contain more explicit options until the desired program is loaded.

Another significant advance in our use of FIRST resulted from a shift from employing floppy disk stand-alone programs to loading the definitions of frequently used words from a shared area on the Corvus disk. To appreciate the advantages of sharing common definitions in a dictionary-based language such as FIRST, it is necessary to distinguish between the FIRST screen editor for program development from file editors employed by most software systems. Programming with FIRST's screen editor involves editing selected screen-size blocks $(1,024$ bytes) of the disk, one at a time, to include the definitions of new words based on existing words either from the core words of FIRST or from previously loaded screens. Since a program is loaded into memory one screen at a time, the various subroutines (words) a program comprises need not be stored on consecutive disk locations under a single file name, as with most file editors. Thus, with FIRST, it is possible to define an extremely large number of words on a disk, but to load only selected words into memory as part of any given program sequence. By obtaining a series of common definitions used in many programs, it became easy to ensure immediate availability of improved versions of frequently used words, since they are now defined in only one location on the Corvus disk. Furthermore, programmers can now refer to an index of screens containing these "common words" to determine which components have already been defined and tested.

Operationally, the $20 \mathrm{M}$ byte Corvus disks have the capacity of 137 single-sided floppy disks, each with 140 screens of information. To index available Corvus disk space, our Apple II/FIRST-Corvus networks format the Corvus disk like a series of floppy disks. Consecutive floppy disk equivalents on the Corvus disk, called volumes, are numbered sequentially from 0 to 136 , and the screens within each volume are numbered sequentially from 0 to 139. We have reserved Volumes 0-19 for programming, and the remainder of the volumes have been allocated for data. Volume 0 contains FIRST words for the menu system, video storage and retrieval, multiple screen copy routines, Corvus-to-Corvus and floppy-toCorvus transfers, and the volume-by-volume directory. In addition, Volume 1 contains the indexed battery of frequently used words, Volume 2 a backup copy of the FIRST source in binary, and Volume 3 a listing of the FIRST Reference Manual. In the remaining program volumes, there are stimulus-control/data-collection, signalanalysis, analysis-of-variance, and statistical-summary programs. Since the output devices available in each of the three conditioning laboratories differ slightly, a separate volume has been designated for their stimuluscontrol/data-collection programs. Each data-collection program ensures access to enough volumes for storage of the digitized response topographies and the extracted dependent variable measures of conditioning for a single conditioning session. Subsequent to each conditioning session, these data are transferred for storage onto video cassettes. Additional volumes are available for those occasions on which the extracted dependent variable measures from a session, multiple sessions, or an entire experiment are restored to the Corvus disk from video cassettes for analysis.

\section{CONCLUSION}

The multinetwork Apple II/FIRST system constitutes a powerful multiuser facility for experimental control, large-scale data acquisition, sophisticated data processing, and long-term data storage. Conversion of separate Apple II/FIRST subsystems to a multinetwork configuration has provided for larger, faster, and more reliable mass storage, more stable and compact long-term data 
storage, greater uniformity in software development based on shared access to common subroutines, and faster, more advanced data-analysis programs for processing extremely large data sets. Furthermore, we have experienced faster turnaround time for large-scale data-processing tasks on our networks than we previously had experienced on larger mainframe facilities, due, in part, to unlimited access time and the ability to reconfigure the networks to accommodate dedicated processing. In our judgment, the full power and flexibility of our Apple II/FIRST multinetwork system has yet to be realized.

\section{REFERENCES}

Harvey, J. A., Gormezano, I., \& Cool-Hauser, V. A. (1983). Effects of scopolamine and methylscopolamine on classical condition- ing of the rabbit nictitating membrane response. Journal of Pharmacology and Experimental Therapeutics, 225, 42-49.

JoHnSON, M. A. (1981). The analysis of multiphasic signals with the Apple II/FIRST microprocessor system. Behavior Research Methods \& Instrumentation, 13, 276-280.

Marshall-Goodell, B., Gormezano, I., SCANDRett, J., \& CaCioppo, J. (1981). The microcomputer in social psychophysiological research: An Apple II/FIRST laboratory. Sociological Methods and Research, 9, 502-512.

Marshall-Goodell, B., Schreurs, B. G., \& Gormezano, I. (1982). Ruler vs. the Apple II/FIRST system analysis of analog signals in classical conditioning. Behavior Research Methods \& Instrumentation, 14, 519-525.

SCANDRETT, J., \& GoRmezano, I. (1980). Microprocessor control and A/D data acquisition in classical conditioning. Behavior Research Methods \& Instrumentation, 12, 120-125.

Schreurs, B., Gormezano, I., \& Harvey, J. A. (1983). Apple II $/$ FIRST system control of electrical brain stimulation in the rabbit. $B e$ havior Research Methods \& Instrumentation, 15, 167-170. 